\title{
Fasting in oncology: a word of caution
}

\author{
Riccardo Caccialanza®, Giuseppe Aprile, Emanuele Cereda and \\ Paolo Pedrazzoli
}

We read with interest the Opinion article by Nencioni and colleagues (Fasting and cancer: molecular mechanisms and clinical application. Nat. Rev. Cancer 18, 707-719 (2018) $)^{1}$ on molecular mechanisms involved in the potential application of fasting in oncology. We accept that fasting and calorie restriction may represent an intriguing springboard for developing future strategies with potential benefits in cancer treatment. However, we would like to point out that the clinical application of fasting and fasting-mimicking diets (FMDs) should only be undertaken with extreme caution, and that the media's enthusiasm for this approach is excessive and unjustified.

According to a recent consensus article, while the results obtained by fasting in cellular and animal models might conceivably be transferred to and benefit patients with cancer in terms of treatment response, toxicity and survival remain to be ascertained ${ }^{2}$.

Several trials have been underway to determine the potential for short-term fasting (STF) in reducing the side effects and enhancing the efficacy of chemotherapy. However, although a considerable number of these have been completed, the results have not yet been published ${ }^{3}$.

Over the past year, only one pilot crossover trial in 34 patients with gynaecological cancer receiving chemotherapy showed that quality of life impairment was lower during STF than in non-fasted periods ${ }^{4}$. Larger studies to prove the effect of STF as an adjunct to chemotherapy have been lacking, and the need for larger studies has been stressed by several reviews published in the past 8 years ${ }^{3,5-10}$.

Also, completed and ongoing clinical trials have only excluded patients with low body mass index and patients reporting significant unintentional weight loss ${ }^{3,11,12}$, which clearly overlooks the issues of malnutrition and sarcopenic obesity and their negative impact on dose-limiting toxicity and survival ${ }^{2,13,14}$. The prevalence of malnutrition and sarcopenia in patients with cancer, which depends on tumour stage and site, may be up to $80 \%{ }^{14}$. Therefore, an established indication for fasting, which could be limited to a very small subset of patients, is not yet available. The statement by Nencioni et al., "We propose that the combination of FMDs with chemotherapy, immunotherapy or other treatments, represents a potentially promising strategy to increase treatment efficacy, prevent resistance acquisition and reduce side effects" ${ }^{\prime}$, is devoid of clear clinical evidence and conflicts with recent recommendations from international scientific societies $^{14,15}$.

Another worrying aspect is that the application of fasting in oncology has been prematurely reported by the media as a potential advance in medical oncology, to the point where FDM kits have recently been commercialized ${ }^{2}$. These may negatively interfere with cancer care, as patients at risk of malnutrition or sarcopenia could autonomously decrease protein-calorie intake during treatment ${ }^{15}$.

Data on fasting and calorie restriction in combination with chemotherapy currently represent only a potential for clinical development, and future articles reviewing this topic can hopefully include conclusive clinical data in order to consider whether fasting can be used in clinical practice.

There is a reply to this letter by Nencioni, A., Caffa, I., Cortellino, S. \& Longo, V. Nat. Rev. Cancer https://doi.org/ 10.1038/s41568-018-0100-x (2019).
Riccardo Caccialanza id ${ }^{1 *}$, Giuseppe Aprile ${ }^{2}$, Emanuele Cereda ${ }^{1}$ and Paolo Pedrazzoli ${ }^{3}$ 'Clinical Nutrition and Dietetics Unit, Fondazione IRCCS Policlinico San Matteo, Pavia, Italy. ${ }^{2}$ Department of Oncology, San Bortolo General Hospital, Vicenza, Italy.

${ }^{3}$ Medical Oncology Unit, Fondazione IRCCS Policlinico San Matteo and Department of Internal Medicine, University of Pavia, Pavia, Italy. *e-mail:r.caccialanza@smatteo.pv.it https://doi.org/10.1038/s41568-018-0098-0

1. Nencioni, A. et al. Fasting and cancer: molecular mechanisms and clinical application. Nat. Rev. Cancer 18, 707-719 (2018)

2. Caccialanza, R. et al. To fast, or not to fast before chemotherapy, that is the question. BMC Cancer 18 , 337 (2018)

3. O'Flanagan, C. H. et al. When less may be more: calorie restriction and response to cancer therapy. BMC Med. 15, 106 (2017).

4. Bauersfeld, S. P. et al. The effects of short-term fasting on quality of life and tolerance to chemotherapy in patients with breast and ovarian cancer: a randomized cross-over pilot study. BMC Cancer 18, 476 (2018).

5. Longo, V. D. \& Fontana, L. Calorie restriction and cancer prevention: metabolic and molecular mechanisms. Trends Pharmacol. Sci. 31, 89-98 (2010).

6. Lee, C. \& Longo, V. D. Fasting versus dietary restriction in cellular protection and cancer treatment: from model organisms to patients. Oncogene 30, 3305-3316 (2011).

7. Lee, C., Raffaghello, L. \& Longo, V. D. Starvation, detoxification, and multidrug resistance in cancer therapy. Drug Resist. Updat. 15, 114-122 (2012).

8. Longo, V. D. \& Mattson, M. P. Fasting: molecular mechanisms and clinical applications. Cell Metab. 19 , 181-192 (2014).

9. Vernieri, C. et al. Targeting cancer metabolism: dietary and pharmacologic interventions. Cancer Discov. 6 1315-1333 (2016).

10. Brandhorst, S. et al. Protective effects of short-term dietary restriction in surgical stress and chemotherapy. Ageing Res. Rev. 39, 68-77 (2017).

11. Dorff, T. B. et al. Safety and feasibility of fasting in combination with platinum-based chemotherapy. BMC Cancer 16, 360 (2016).

12. de Groot, S. et al. The effects of short-term fasting on tolerance to (neo) adjuvant chemotherapy in HER2negative breast cancer patients: a randomized pilot study. BMC Cancer 15, 652 (2015)

13. Carneiro, I. P., Mazurak, V. C. \& Prado, C. M. Clinical implications of sarcopenic obesity in cancer Curr. Oncol. Rep. 18, 62 (2016).

14. Arends, J. et al. ESPEN guidelines on nutrition in cancer patients. Clin. Nutr. 36, 11-48 (2017).

15. Caccialanza, R. et al. Nutritional support for cancer patients: still a neglected right? Support. Care Cancer 25, 3001-3004 (2017).

Acknowledgements

The authors gratefully thank P. Baines for assistance in editing the manuscript.

\section{Author contributions}

R.C. researched data for the article, contributed to the discussion of content, wrote and edited the manuscript before submission. G.A. and P.P. contributed to the discussion of content, wrote and edited the manuscript before submission. E.C. researched data for the article, contributed to the discussion of content, and edited the manuscript before submission.

\section{Competing interests}

The authors declare no competing interests.

\section{Publisher's note}

Springer Nature remains neutral with regard to jurisdictional claims in published maps and institutional affiliations. 